\title{
Avaliação do desempenho escolar de crianças contaminadas por chumbo
}

\author{
Olga Maria Piazentin Rolim Rodrigues \\ Universidade Estadual Paulista de Bauru - SP \\ Christiana Gonçalves Meira de Almeida \\ Faculdades Integradas de Ourinhos - SP \\ Veronica Aparecida Pereira \\ Universidade Federal da Grande Dourados - MS \\ Vera Lúcia Messias Fialho Capellini \\ Universidade Estadual Paulista de Bauru - SP
}

\begin{abstract}
Resumo
A contaminação de crianças por chumbo tem sido objeto de investigação no âmbito da saúde pública. Neste estudo avaliou-se o desempenho acadêmico de 102 crianças do Ensino Fundamental. Os participantes foram divididos em dois grupos: um com 34 crianças não contaminadas ou com nível inferior a $5 \mu \mathrm{g} / \mathrm{dl}$ e o outro, de 68 crianças, estava com nível de contaminação por chumbo no sangue entre 10 and $40 \mu \mathrm{gg} / \mathrm{dl}$. Para avaliação foram utilizados o Teste de Desempenho Escolar (TDE) e anamnese. Os resultados indicaram melhor desempenho acadêmico para o segundo grupo, com diferenças significantes em aritmética, leitura e desempenho geral. A comparação entre os sexos indicou melhor desempenho para meninas, dado coerente com a percepção dos pais na anamnese. Embora outras variáveis estejam presentes, os dados apontam prejuízos acadêmicos maiores para crianças com índices de chumbo mais altos. Esses achados requerem políticas públicas para controle da contaminação e redução dos danos em contaminados.
\end{abstract}

Palavras-chave: Teste; rendimento escolar; saúde pública.

\section{Academic performance evaluation of lead-poisoned children}

\begin{abstract}
The lead contamination in children has been the subject of research in the field of public health. This study evaluated the academic performance of 102 children from first to fourth grade. The subjects were divided into two groups. The first group was composed of 34 children without lead poisoning or with poison at levels lower than $5 \mu \mathrm{g} / \mathrm{dl}$. The second group was composed of 68 children with blood lead levels between 10 and $40 \mu \mathrm{g} /$ dl. The instruments used to evaluate the academic performance were anamnesis and a scholarly performance test called Teste de Desempenho Escolar, TDE. The results indicated better academic performances from the second group with significant differences in arithmetic, reading and general scores. In a comparison between genders, the girls had better performances than the boys. These results were consistent with the parents' perception in anamnesis. Although other variables were present, the data showed great academic damage for children with higher leadpoison. These outcomes require political policies to control contamination and intervention in this population.
\end{abstract}

Descriptors: Academic Performance Test; School performance; public health

\section{Evaluación del desempeño escolar de niños contaminadas por plomo}

\begin{abstract}
Resumen
La contaminación de niños por plomo ha sido objeto de investigación en el ámbito de la salud pública. En este estudio se evaluó el desempeño académico de 102 niños de la Enseñanza Fundamental. Los participantes fueron divididos en dos grupos: uno con 34 niños no contaminados o con nivel inferior a $5 \mu \mathrm{g} / \mathrm{dl}$ y el otro, de 68 niños, estaba con nivel de contaminación por plomo en la sangre entre 10 y $40 \mu \mathrm{g} / \mathrm{dl}$. Para evaluación fueron utilizados el Test de Desempeño Escolar (TDE) y anamnesis. Los resultados indicaron mejor desempeño académico para el segundo grupo, con diferencias significantes en aritmética, lectura y desempeño general. La comparación entre los sexos indicó mejor desempeño para niñas, dato coherente con la percepción de los padres en la anamnesis. Aunque otras variables estén presentes, los datos apuntan perjuicios académicos mayores para niños con índices de plomo más altos. Esas descubiertas requieren políticas públicas para control de la contaminación y reducción de los daños en contaminados.
\end{abstract}

Palabras-clave: Test; aprovechamiento escolar; salud pública. 


\section{Introdução}

O chumbo é o mineral que mais causa intoxicações. Está presente no solo, no ar e na água. As principais fontes são a indústria e reciclagem de baterias automotivas, chapas de metal semiacabado, canos de metal, aditivos em gasolina, munição, tintas, soldas de latas, pesticidas, agrotóxicos e outras. (Meireles,1981).

A toxicidade do chumbo gera efeitos clínicos - às vezes sutis - ou bioquímicos que acabam por envolver vários sistemas de órgãos e atividades bioquímicas. Estudos com ratos demonstram que a exposição crônica ao chumbo acarreta problemas de memória e aprendizagem, dentre outros prejuízos (Zhang, \& Liu, 2010; Diao, Wu, \& Zhu, 2011). Em crianças, os efeitos críticos atingem o sistema nervoso, enquanto em adultos, a exposição ocupacional excessiva, ou mesmo acidental, acarreta tais efeitos na neuropatia periférica e na nefropatia crônica (Thacker, Hoffman, Smith, Steinberg, \& Zack, 1992; Banks, Ferretti, \& Shucard, 1997; Araújo, Pivetta, \& Moreira, 1999; Malta, Trigo, \& Cunha, 2000; Moreira, \& Moreira, 2004; Mahmoudian, Modaresi, Zarei, Poursafa, \& Kelishadi, 2009).

Problemas com a contaminação por chumbo em humanos estão presentes em diversas partes do mundo. Nos países em desenvolvimento, o segmento industrial responsável pelo maior consumo de chumbo é a produção de baterias chumbo-ácidas. Essas baterias são fabricadas mediante uma tecnologia bastante simples, podendo ser realizada em pequena escala, o que a torna atraente para esses países. Em razão das propriedades tóxicas do chumbo e das condições de trabalho prevalentes na maioria dessas indústrias, os trabalhadores desse setor encontram-se frequentemente expostos a elevadas concentrações desse elemento e, consequentemente, sujeitos a intoxicação (Araújo, \& cols., 1999). Não incomum é o estabelecimento de moradias populares próximas aos setores de produção, estendendo-se os riscos de contaminação à família, principalmente às crianças, uma vez que são mais vulneráveis.

Um estudo que avaliou os níveis de contaminação por chumbo em Chengdu, na China; apontou que $8,21 \%$ das crianças com menos de sete anos tinham altos níveis de contaminação por chumbo. Esses resultados foram atribuídos a questões específicas da vida dessas pessoas, entre elas, hábitos alimentares e padrões de higiene (Jiang, \& cols., 2010). A implementação de políticas públicas, como descrito no estudo de He, Wang e Zhang (2009), pode contribuir para a diminuição de níveis de contaminação por chumbo em crianças, como apontou o estudo em relação às crianças chinesas a partir do ano de 2004 , com normas mais seguras do processo de produção.

Redes de notícias internacionais descreveram que os níveis de contaminação por chumbo na Nigéria não têm precedentes na história e levam à urgência de medidas efetivas para redução da contaminação e de assistência à população afetada (Moszynski, 2010). Choudhari, \& cols. (2010) apontaram a necessidade de medidas efetivas no combate à contaminação de crianças que residem perto de minas de zinco e chumbo.

De acordo com o Committee on Environmental Health (2005) nos Estados Unidos, apesar das medidas tomadas em relação ao risco de contaminação, ainda se verificou que, nesse período, cerca de $25 \%$ das crianças vivem em casas pintadas com tintas que contêm chumbo em sua composição. Isto levou à instauração de políticas de prevenção e projetos com o objetivo de reformar as casas para diminuir os riscos de danos para a saúde dessas crianças.

O Center for Control and Prevention apontou o índice de $10 \mu \mathrm{g} / \mathrm{dl}$ de sangue como limiar de segurança para a saúde infantil, sendo os índices superiores capazes de ocasionar danos à saúde (Jain, \& cols., 2005). O índice mínimo foi estabelecido levando-se em conta que seria praticamente impossível alguém ser totalmente isento da exposição ao chumbo, em razão de sua presença em diversos produtos da atualidade (enlatados, baterias, cosméticos entre outros); contudo, pesquisas conduzidas pelo Committee on Environmental Health apontam que mesmo concentrações de chumbo abaixo de $10 \mu \mathrm{g} / \mathrm{dl}$ de sangue podem prejudicar processos cognitivos. Preventivamente, seria oportuno que todas as crianças se submetessem a exames anuais para detectar possíveis contaminações, no entanto esta prática ainda não é corrente na maioria dos países. Os exames são conduzidos, muitas vezes, na iminência de altos índices de exposição.

Apesar das medidas adotadas nos Estados Unidos para controle da contaminação de crianças por chumbo, como a retirada do chumbo da pintura e da gasolina em 1970, terem contribuído para diminuírem os níveis de contaminação, ainda se observou de 310 mil crianças com menos de cinco anos têm níveis elevados de chumbo no sangue (Warniment, Tsang, \& Galazka, 2010). Essas investigações indicam que o problema da contaminação por chumbo não se restringe a países subdesenvolvidos.

Os efeitos da absorção de chumbo variam com a idade. Nos sintomas iniciais, reversíveis, destacam-se dores abdominais, perda de apetite, constipação intestinal, fadiga, distúrbios do sono e diminuição das atividades físicas (Stiles, \& Bellinger, 1993). Pela falta de exames clínicos, os sintomas físicos podem ser confundidos com uma série de outros problemas de saúde.

Bellinger $(1995,2008)$ afirmou que mesmo quantidades relativamente pequenas de chumbo podem causar rebaixamento permanente da inteligência em crianças, resultando em prejuízos acadêmicos e distúrbios psicológicos. Thacker, \& cols. (1992) apontaram que, em geral, o público mais afetado vive também em áreas mais pobres, estando exposto, além do chumbo, a condições menos favorecidas de vida, o que dificulta ainda mais o diagnóstico.

Muitas pesquisas foram feitas nos últimos 30 anos para avaliar as concentrações de chumbo no sangue e seus efeitos (Yule, \& Lannsdown, 1983; Needleman, \& Gatsonis, 1990; Bellinger, 1995; Tomita, Padula, \& Gepiccb, 2005; Jorge, \& cols., 2008). Os autores indicam como sintomas observáveis em crianças fácil irritabilidade, choro sem causa 
aparente, agitação, baixo rendimento escolar, alterações da memória e anemia. Há consenso também quanto à vulnerabilidade de acrianças aos efeitos do chumbo, devida a vários fatores, como: 1-) as crianças o absorvem mais que os adultos; 2) Crianças têm o hábito de colocar frequentemente na boca objetos que levam sujeiras do solo; 3- como crianças pequenas estão em desenvolvimento rápido e constante, seus sistemas não estão completamente desenvolvidos; 4) mesmo quantidades relativamente pequenas podem causar distúrbios psicológicos e déficits cognitivos.

Apesar da indicação de níveis de chumbo seguros para o organismo infantil, há estudos associados aos baixos níveis de chumbo que descrevem comprometimento do comportamento emocional, da inteligência e da aprendizagem de crianças (Needleman, 1979; Thacker \& cols. 1992; Yule, \& Lansdown, 1983). Os autores mostraram que, embora altos níveis de chumbo tenham efeitos neurotóxicos, ainda há dúvida quanto ao nível em que os efeitos da neurotoxina funcional são atribuídos ao aparecimento do chumbo. Efeitos adversos tais como, por exemplo, redução no desempenho escolar, parecem ocorrer em níveis abaixo de $10 \mu \mathrm{g} / \mathrm{dl}$ de sangue (Belinger, 2008; Needleman, 2009).

O estudo de Stiles e Bellinger (1993) mostrou correlação entre o nível de contaminação por chumbo do sangue de crianças e o vocabulário e a compreensão, quando submetidos ao WISC. O desempenho cognitivo mostrou-se também afetado quando as crianças tinham longo período de exposição, mesmo que com índices baixos (Thomson, \& cols., 1989). De modo semelhante, Miranda Maxson e Kim (2010) constataram que a contaminação por chumbo durante a primeira infância influencia significativamente a probabilidade de problemas cognitivos.

Há algumas evidências de que altos níveis de chumbo em crianças pequenas podem estar associados com maior risco para o Transtorno de Déficit de Atenção e Hiperatividade (TDAH). Esta relação parece mais importante quando a exposição ao chumbo funciona como um irritante no cérebro, prejudicando-o (Benczik, 2000). No estudo de Smith (1985) baixos níveis de chumbo estavam correlacionados a déficits leves em algumas medidas psicométricas, porém havia outros fatores que poderiam interferir, tais como, ambientes desprovidos de condições que propiciassem aprendizagem, pobreza e falta de estímulo dos pais.

O estudo do impacto do chumbo na saúde humana e no ambiente é determinante para a prevenção de intoxicações na população, que estão associadas ao crescente desenvolvimento industrial e tecnológico, principalmente em países em desenvolvimento, onde os riscos são pouco avaliados e controlados. No Brasil, a carência de estudos e de um banco de informações sobre a exposição à contaminação por chumbo, principalmente em relação a crianças, torna necessários projetos que possam subsidiar programas de ação preventiva e de proteção da saúde física e mental desta população. Por essas e outras razões, a intoxicação por chumbo tem sido um problema internacional de saúde pública.
Com relação aos efeitos do contexto socioeconômico, Campana (1985) estudou 536 crianças escolares em Botucatu em duas escolas públicas, sendo uma do centro e outra da periferia. Como resultado constatou um nível intelectual insatisfatório de $17,53 \%$ considerando-se as duas escolas, e no tocante apenas à escola da periferia, o índice subiu para e de $28,99 \%$. Entre as crianças com desempenho inferior, a quase totalidade pertencia ao nível socioeconômico mais baixo. Resultados semelhantes também foram apontados nos estudos de Macedo, Andreucci e Montelli (2004) e Miranda e cols. (2010).

Duncan, Brooks-Gunn e Klebanov (1994), ao estudarem crianças de cinco anos de idade considerando a renda familiar e a escolaridade materna constataram que havia relação direta entre essas duas variáveis e o desempenho escolar da amostra: quanto mais baixa a renda e menor a escolaridade materna, pior o desempenho cognitivo das crianças.

Esses resultados devem ser interpretados em seu contexto, para que não se compreendam as dificuldades escolares como inerentes à criança de nível socioeconômico mais baixo. Collares e Moyses (1996) mostraram que o cotidiano escolar é repleto de preconceitos e juízos prévios sobre os alunos, de modo que, crianças pobres, negras e/ou moradoras da zona rural são culpabilizadas por não aprenderem e, a escola, apresenta-se como impossibilitada de lidar com uma clientela aparentemente despreparada para aprender.

Sobre o ambiente familiar, Debaryshe, Patterson e Capaldi (1993) apontaram que o envolvimento adequado dos pais com a escolaridade dos filhos promove não só o envolvimento dos filhos no processo de aprendizagem, mas também um melhor rendimento escolar e bom nível de autoestima. Também Ginsburg e Bronstein, (1993) relataram que a valorização, o engajamento e o encorajamento dos pais com relação à escolaridade dos filhos promovem nestes a motivação intrínseca para o aprendizado e parecem associados ao maior grau de iniciativa dos filhos em sala de aula. Em contrapartida, pais que não se envolvem, não se engajam na escolaridade dos filhos ou os cobram de maneira excessiva geram um baixo nível de motivação intrínseca e um alto nível de motivação extrínseca, de forma que esses adolescentes têm baixa iniciativa em sala de aula.

Dascanio e Do Valle (2007) apontaram que, como a contaminação por chumbo está relacionada a alterações comportamentais, há possibilidade de essas alterações influenciarem a forma de interação entre pais e filhos. Diante disso, ressaltaram a importância de desenvolver estudos no sentido de compreender os efeitos do chumbo no comportamento infantil e seu impacto no relacionamento familiar.

$\mathrm{Na}$ família, na escola e em todo o contexto social existe uma série de valores que permeiam a concepção de aprendizagem, escola e desempenho acadêmico de crianças. Dessa forma é pertinente investigar diferenças de gênero e suas relações com o desempenho acadêmico. Como afirma Carvalho $(2001,2003)$, este tema é vastamente discutido na literatura em resultados de inúmeras pesquisas. 
Dentre outras questões, seus estudos buscaram compreender os processos que conduziram um maior número de meninos a obterem conceitos negativos e a serem mais indicados para atividades de recuperação. Em relação ao gênero dos alunos, percebeu-se que este é um fator decisivo para os problemas relacionados ao desempenho escolar e ao merecimento de elogios por parte dos professores. A variável diferença de gênero apresentou-se de forma mais marcante do que a renda tomada isoladamente. Num outro dado encontrado, os meninos foram os que apresentavam mais problemas de comportamento, eram tidos como mais indisciplinados, punidos com advertências e suspensões, na grande maioria das vezes por agressões físicas e verbais a colegas.

No estudo de Carvalho (2001), compreendeu-se que as atitudes "desordeiras" dos meninos fossem a base principal para os problemas escolares que estes enfrentavam (incluídas as indicações para o reforço), mais do que propriamente dificuldades de aprendizagem. O autor constatou uma sensível diferença em favor das meninas no desempenho escolar, principalmente em Português ou Linguagem. Já em Matemática, os grupos se apresentam iguais, embora se diferenciem em favor dos meninos à medida em que estes ascendem no sistema escolar, fato exemplificado a partir dos resultados do SAEB de 1999 (Instituto Nacional De Estudos Pedagógicos, 2001); entretanto pouco se tem avançado em termos de explicações para o fenômeno e os meninos predominam largamente entre os indicados para o reforço, em todos os grupos de raça e faixas de renda.

O relatório publicado pela UNICEF (Bellamy, 2004) apontou que o fenômeno do fracasso escolar entre meninos é, há algumas décadas, uma característica da maioria dos países latino-americanos e do Caribe. Esse mesmo texto ressalta que essa desigualdade entre gêneros não é um fenômeno simples, mas sim, um tema no qual fatores relacionados com o gênero se combinam com questões raciais e de classe. Até recentemente esse fenômeno era considerado comum apenas em países industrializados e eram muitas vezes explicados com base na hipótese de que os meninos eram obrigados a trabalhar mais cedo tendo que sustentar a família.

Embora o objeto de estudo de Correa e Mac Lean (1999) tenha sido a análise das narrativas produzidas pelas crianças acerca da aprendizagem inicial da leitura e da escrita, a primeira informação relevante foi a diferença de gênero associada ao sucesso ou fracasso escolar dessas crianças na alfabetização. Um maior número de meninas do que de meninos é promovido para a segunda série. Os que não conseguiram alcançar a promoção para a série seguinte são, em maior número, os meninos.

Desse modo, é importante compreender o fracasso escolar como síntese de múltiplas determinações (Meira, 2003), uma das quais pode ser a contaminação por chumbo. Considerando-se a dificuldade de isolar o fator contaminação por chumbo de outros fatores que influenciam no desenvolvimento escolar, esta variável não deve ser com- preendida de maneira isolada, mas deve ser avaliada dentro do contexto no qual a criança está inserida.

Para investigação de problemas escolares, os testes psicométricos podem ser importantes instrumentos de avaliação. O Teste de Desempenho Escolar (TDE) tem sido usado para avaliar o desempenho acadêmico de crianças em diversas condições. Dias, Enumo e Azevedo (2004) utilizaram o TDE para investigar os efeitos de um programa de criatividade sobre o desempenho acadêmico e cognitivo de 17 alunos com dificuldade de aprendizagem, da $2^{a}$ e $3^{a}$ séries do Ensino Fundamental público de Vitória/ES, comparando-os a um grupo-controle. Os resultados indicaram uma melhora significativa no desempenho acadêmico do grupo que participou do treinamento.

A contaminação por chumbo também pode ser um fator incluído no grupo de fatores que pode influenciar o desempenho acadêmico das crianças. A esse respeito Bellinger (2008) afirma que mesmo quantidades relativamente pequenas de chumbo podem causar rebaixamento permanente da inteligência em crianças, resultando em prejuízos acadêmicos e distúrbios psicológicos. Como o TDE, analisado em diversas pesquisas, obteve parecer favorável no Sistema de Avaliação de Testes Psicológicos do Conselho Federal de Psicologia e sendo usado em diversas pesquisas, ele pode ser um instrumento eficaz de análise em investigação das relações entre dificuldades acadêmicas e contaminação por chumbo.

Capellini, Rodrigues, Melchiori e Valle (2008) utilizaram o Teste de Desempenho Escolar - TDE (Stein, 1994) na avaliação do rendimento escolar de crianças contaminadas por chumbo, em comparação com crianças não contaminadas. Participaram três grupos com 25 crianças cada, de seis a treze anos. O grupo 1 constituía-se de crianças contaminadas por chumbo; o Grupo 2, de crianças com chumbo zero comprovado, embora todos residissem no local da contaminação; e o grupo 3, de crianças de um bairro com características sociodemográficas semelhantes às do local da contaminação, porém, sem contaminação comprovada. Os resultados mostraram melhor desempenho para o grupo de crianças não contaminadas (grupo 2) e pior para o grupo contaminado (grupo 1). O grupo 3 obteve, em média, índices maiores que os demais grupos. Para os três grupos, o melhor desempenho foi observado no subteste de leitura e o pior em aritmética.

Apesar da escassez de descrições na literatura especializada brasileira acerca dos efeitos no rendimento acadêmico decorrente da contaminação por chumbo, as informações disponíveis apontam uma incidência relativamente alta desses casos.

A contaminação por chumbo na cidade de Bauru foi notificada pela Companhia de Tecnologia de Saneamento Ambiental (Cetesb) à Secretaria Municipal de Saúde em janeiro de 2002, no local onde se instalara uma fábrica de acumuladores (baterias) desde 1958, em uma área periférica do município. Dejetos com sal de óxido de chumbo e sulfato de chumbo estavam presentes na poeira (pluma) e a 
deposição de chumbo metálico no solo foi detectada (Instituto Ambiental Vidágua, 2002).

Nas proximidades da fábrica ( $1.000 \mathrm{~m}$ da fonte) foram identificadas 314 crianças com taxas de plumbemia superiores àquelas aceitáveis pela Organização Mundial de Saúde (10 $\mathrm{\mu g} / \mathrm{dl}$ sangue - 10 microgramas por decilitro de sangue). Em virtude desse fato, foram desencadeadas ações emergenciais, com o objetivo de reduzir riscos de recontaminação, que incluíram a raspagem de camada superficial das vias públicas, resultando em $1.392 \mathrm{~m}^{3}$ de terra com material tóxico, que permanece depositada nas dependências da fábrica. Foi promovida a aspiração de poeira do interior de 164 residências, com a utilização de equipamento industrial, bem como a lavagem e vedação das caixas de água (Tomita, \& cols., 2005).

Diante da necessidade de participação dos serviços públicos e de seus gestores, com apoio de instituições de ensino e pesquisa, foram envolvidos profissionais da SES/ SP, SMS-Bauru, Cetesb, Ministério da Saúde (MS), Fundação Jorge Duprat Figueiredo de Segurança e Medicina do Trabalho (Fundacentro), Centro de Intoxicações da Faculdade de Ciências Médicas da Universidade Estadual de Campinas (FCM-Unicamp), Faculdade de Medicina de Botucatu da Universidade Estadual Paulista Júlio Mesquita Filho (FMB-UNESP), Faculdade de Ciências (FC-Unesp/Bauru), Faculdade de Odontologia de Bauru da Universidade de São Paulo (FOB-USP) e Hospital de Reabilitação de Anomalias Craniofaciais (HRAC-USP). Seus representantes compõem o Grupo de Estudo e Pesquisa da Intoxicação por Chumbo em Crianças de Bauru (GEPICCB), que desencadeou medidas de investigação clínico-epidemiológica e tratamento das crianças.

Na tentativa de contribuir para o entendimento, o controle e a prevenção com o objetivo de minimizar riscos de deficiência ou de problemas escolares posteriores, este estudo foi proposto, visando avaliar o desempenho acadêmico de crianças contaminadas por chumbo. Busca-se aqui des- crever e comparar o desempenho acadêmico de crianças contaminadas por chumbo com crianças sem contaminação comprovada, considerando-se as variáveis contaminação por chumbo e sexo.

\section{Método}

O presente estudo foi aprovado pelo Comitê de Ética da Faculdade de Ciências/UNESP, Bauru, atendendo às normas preestabelecidas pelo CONEP (1996).

Foram avaliadas 34 crianças, das quais 14 apresentavam níveis de chumbo inferiores a $5 \mathrm{mg} / \mathrm{dl}$ de sangue moradoras na área de contaminação e 20 crianças sem contaminação comprovada, oriundas de um bairro distante cerca de 15 quilômetros da área de contaminação, em um bairro estritamente residencial (grupo 1). O grupo 2 foi composto por 68 crianças, com contaminação comprovada (níveis de chumbo entre 10 e $40 \mathrm{mg} / \mathrm{dl}$ de chumbo no sangue), sendo 37 meninos e 30 meninas. Os bairros dos dois grupos se assemelham em termos de condições socioeconômicas.

Para a entrevista com os pais foi utilizada uma anamnese contendo informações demográficas dos participantes e dados sobre a história escolar passada e atual da criança.

Para a avaliação do desempenho acadêmico foi utilizado o Teste de Desempenho Escolar (TDE) (Stein, 1994), folhas sulfite, lápis preto e borracha. O TDE é um instrumento psicométrico que busca oferecer, de forma objetiva, uma avaliação das capacidades para o desempenho escolar, mais especificamente da escrita, aritmética e leitura. $O$ teste foi concebido para a avaliação de escolares de $1^{a}$ a $6^{a}$ séries do Ensino Fundamental. É composto por três subtestes: de escrita, de aritmética e de leitura. O teste prevê, para a primeira série, a classificação em quatro categorias: Inferior; Médio inferior; Médio superior e Superior. Para as demais séries são previstas três classificações: inferior; médio e superior.

Tabela 1. Desempenho no TDE

\begin{tabular}{|l|c|c|c|c|c|}
\hline \multirow{2}{*}{$\begin{array}{l}\text { Áreas } \\
\text { avaliadas }\end{array}$} & \multicolumn{3}{|c|}{ Médias e desvio padrão obtidos os índices classificatórios do TDE } \\
Avaliação Geral \\
\cline { 2 - 5 }
\end{tabular}

*significativo para o grau de confiabilidade $>95 \%$; ** significativo para o grau de confiabilidade $>90 \%$.

G1 - Alunos com índices abaixo de $5 \mu \mathrm{g}$ de pb/dl de sangue ou fora da área de contaminação; G2: Alunos com índices acima de $10 \mu \mathrm{g}$ de $\mathrm{pb} / \mathrm{dl}$ de sangue. 
Para o presente estudo foi utilizada a classificação proposta por Capellini (2001), utilizando-se a subdivisão do nível inferior do TDE em três subclasses: Inferior/Inferior; Inferior/Médio; Inferior/Superior.

Os participantes do Grupo 1 foram avaliados no CPA e os participantes do Grupo 2, nas suas respectivas escolas. Para compor o Grupo 2, crianças das mesmas séries das do Grupo 1 foram convidadas a participar do projeto e levaram para casa o Termo de Consentimento Livre e Esclarecido.

Para a análise dos dados utilizou-se o teste $t$ de Student para comparação das médias entre grupos. Para a correlação entre variáveis níveis de chumbo e desempenho no TDE utilizou-se o teste de Spearmann.

\section{Resultados}

O desempenho acadêmico das crianças dos grupos 1 e 2 foi comparado considerando-se as variáveis nível de contaminação e sexo. Na tabela 1 são apresentados os resultados do desempenho no TDE nas áreas de Escrita, Aritmética, Leitura e Desempenho Geral dos Grupos 1 e 2.

O desempenho no TDE foi melhor no Grupo 1 em todas as áreas, apresentando níveis de significância para as áreas de aritmética, leitura e desempenho geral.

Considerando-se o desempenho entre os sexos, na avaliação intergrupos, conforme observado nas tabelas 2 e 3 , os resultados das médias continuam maiores no grupo 1 , tanto para meninas quanto para meninos. Neste recorte, os níveis de significância intergrupos foram observados na área de leitura e desempenho Geral.

Para identificar possíveis diferenças entre participantes de ambos os grupos em relação às diferenças de sexo, foram pareados os resultados de desempenho no TDE do grupo 1 (meninas $x$ meninos) conforme tabela 4 e grupo 2 (meninas $x$ meninos) conforme tabela 5 .

A média obtida no TDE é maior para as meninas no Grupo 1, porém as diferenças não se mostraram significantes.

Tabela 2. Comparação do desempenho de participantes do sexo feminino dos Grupos 1 e 2.

\begin{tabular}{|c|c|c|c|c|c|}
\hline \multirow{3}{*}{$\begin{array}{l}\text { Áreas } \\
\text { avaliadas }\end{array}$} & \multicolumn{4}{|c|}{$\begin{array}{l}\text { Médias e desvio padrão obtidos os índices classificatórios do TDE } \\
\text { Avaliação Intergrupos - Gênero feminino }\end{array}$} & \multirow{3}{*}{$p$} \\
\hline & \multicolumn{2}{|c|}{ Grupo 1} & \multicolumn{2}{|c|}{ Grupo 2} & \\
\hline & Média & Desvio Padrão & Média & Desvio Padrão & \\
\hline Escrita & 3,0625 & 1,56924 & 2,94 & 1,340 & ,835 \\
\hline Aritmética & 3,5625 & 0,72744 & 3,38 & 1,025 & ,580 \\
\hline Leitura & 3,53 & 1,586 & 2,7647 & 1,43742 & ,223 \\
\hline Total & 3,1176 & 1,40900 & 3,35 & 1,115 & , 595 \\
\hline
\end{tabular}

*significativo para o grau de confiabilidade $>95 \%$; ${ }^{* *}$ significativo para o grau de confiabilidade $>90 \%$. G1 - Alunas com índices abaixo de $5 \mu \mathrm{g}$ de pb/dl de sangue ou fora da área de contaminação; G2: Alunas com índices acima de $10 \mu \mathrm{g}$ de $\mathrm{pb} / \mathrm{dl}$ de sangue.

Tabela 3. Comparação do desempenho de participantes do sexo masculino dos Grupos 1 e 2.

\begin{tabular}{|c|c|c|c|c|c|}
\hline \multirow{3}{*}{ Áreas avaliadas } & \multicolumn{4}{|c|}{$\begin{array}{l}\text { Médias e desvio padrão obtidos os índices classificatórios do } \\
\text { TDE } \\
\text { Avaliação intergrupos - gênero masculino }\end{array}$} & \multirow{3}{*}{$p$} \\
\hline & \multicolumn{2}{|c|}{ Grupo 1} & \multicolumn{2}{|c|}{ Grupo 2} & \\
\hline & Média & Desvio Padrão & Média & Desvio Padrão & \\
\hline Escrita & 3,10 & 1,619 & 2,3000 & 1,45458 & , 138 \\
\hline Aritmética & 3,50 & 1,100 & 2,9500 & 1,43178 & ,225 \\
\hline Leitura & 3,50 & 1,357 & 2,1500 & 1,38697 & ,005 \\
\hline Total & 3,35 & 1,268 & 2,1000 & 1,37267 & ,007 \\
\hline
\end{tabular}

*significativo para o grau de confiabilidade $>95 \%$; ** significativo para o grau de confiabilidade $>90 \%$. G1 - Alunos com índices abaixo de $5 \mu \mathrm{g}$ de $\mathrm{pb} / \mathrm{dl}$ de sangue ou fora da área de contaminação; G2: Alunos com índices acima de $10 \mu \mathrm{g}$ de $\mathrm{pb} / \mathrm{dl}$ de sangue. 
Tabela 4. Resultado da comparação do resultado obtido no TDE entre meninos e meninas do Grupo 1.

\begin{tabular}{|c|c|c|c|c|c|}
\hline \multirow{3}{*}{$\begin{array}{l}\text { Áreas } \\
\text { avaliadas }\end{array}$} & \multicolumn{4}{|c|}{$\begin{array}{l}\text { Médias e desvio padrão obtidos os índices classificatórios do } \\
\text { TDE } \\
\text { Avaliação Intergrupos }\end{array}$} & \multirow{3}{*}{$P$} \\
\hline & \multicolumn{2}{|c|}{ Meninos } & \multicolumn{2}{|c|}{ Meninas } & \\
\hline & Média & Desvio Padrão & Média & Desvio Padrão & \\
\hline Escrita & 2,94 & 1,67 & 3,06 & 1,39 & 0,787 \\
\hline Aritmética & 3,52 & 1,17 & 3,29 & 1,04 & 0,533 \\
\hline Leitura & 3,41 & 1,41 & 3,53 & 1,58 & 0,833 \\
\hline Total & 3,35 & 1,36 & 3,35 & 1,11 & 1,000 \\
\hline
\end{tabular}

Tabela 5. Resultado da comparação do resultado obtido no TDE entre meninos e meninas do Grupo 2.

\begin{tabular}{|c|c|c|c|c|c|}
\hline \multirow{3}{*}{$\begin{array}{l}\text { Áreas } \\
\text { avaliadas }\end{array}$} & \multicolumn{4}{|c|}{$\begin{array}{l}\text { Médias e desvio padrão obtidos os índices classificatórios do } \\
\text { TDE } \\
\text { Avaliação Intergrupos }\end{array}$} & \multirow{3}{*}{$P$} \\
\hline & \multicolumn{2}{|c|}{ Meninos } & \multicolumn{2}{|c|}{ Meninas } & \\
\hline & Média & Desvio Padrão & Média & Desvio Padrão & \\
\hline Escrita & 2,55 & 1,47 & 3,17 & 1,36 & 0,104 \\
\hline Aritmética & 3,1 & 1,29 & 3,31 & 1,10 & 0,537 \\
\hline Leitura & 2,4 & 1,35 & 3,07 & 1,38 & 0,101 \\
\hline Total & 2,4 & 1,45 & 3,20 & 1,27 & 0,060 \\
\hline
\end{tabular}

A média obtida no TDE é maior para as meninas no Grupo 2, apontando índices de significância no desempenho geral.

\section{Discussão}

Como as comparações entre os grupos 1 e 2 indicaram resultados inferiores em todas as áreas para o grupo 2 (com maior índice de contaminação), os dados confirmam os apontamentos sobre prejuízos à saúde e desenvolvimento infantil, indicando relações entre a contaminação por chumbo e dificuldades de aprendizagem.

A adaptação da avaliação do TDE, proposta por Capellini (2001), possibilitou uma análise mais refinada do desempenho no teste, tornando possível a identificação de diferenças mais sutis. Segundo a proposta da autora, o TDE tem 5 níveis (1. Inferior inferior, 2. Inferior médio, 3. Inferior superior, 4. Médio, 5. Superior). Como as médias do grupo 1 estiveram pouco acima de 3 , eles estariam entre o nível Inferior superior e médio, ao passo que o grupo 2 estaria entre o Inferior médio e superior. Isto indica que a transição é muito sutil e que, na versão original do teste, a grande maioria estaria no nível inferior, considerando-se apenas os três níveis propostos por Stein (1994) (inferior, médio e superior). O fato de o desempenho Inferior estar presente em primazia para todas as crianças é um resultado coerente com a literatura, a qual indica que o chumbo pode ser um importante fator nos distúrbios da aprendizagem, mesmo quando os níveis são inferiores ao limiar de segurança (Yule, \& Lansdown, 1983; Needleman, \& Gatsonis, 1990; Bellinger, 1995, 2008, Needleman, 2009). Uma vez que as crianças eram oriundas de um mesmo bairro e tinham condições socioeconômicas muito semelhantes, o fator contaminação apresenta-se como uma variável significativa. Semelhantemente aos estudos de Smith (1985), todas as crianças avaliadas possuem condições socioeconômicas baixas. Nessa amostra o ambiente desfavorável pode não apenas dificultar a aprendizagem, mas também contribuir para que a contaminação por chumbo (passar muitas horas em contato com água, poeira e alimentos contaminados) atinja maiores níveis, ocasionando maiores danos.

Em relação às diferenças entre gêneros percebe-se que o grupo feminino teve um desempenho melhor no Teste de Desempenho Escolar. Este resultado também é descrito por outros pesquisadores (Carvalho, 2001, 2003, 2004), que apontam que as meninas apresentam desempenho acadêmico melhor que o dos meninos. Nos estudos de Carvalho 
(2001) foi constatada uma sensível diferença em favor das meninas no desempenho escolar, principalmente em Português ou Linguagem; já em Matemática nota-se um desempenho semelhante entre os dois sexos, fato exemplificado a partir dos resultados do SAEB de 1999 (Instituto Nacional de Estudos Pedagógicos, 2001). No presente estudo, embora as médias do sexo feminino tenham sido melhores em todas as áreas, a significância foi apontada apenas no desempenho geral do grupo 2 .

É importante ressaltar que as meninas tiveram menores níveis de contaminação por chumbo. Diante disso, faz-se necessário compreender que essas diferenças entre gênero nos resultados no TDE podem estar relacionadas ao nível de contaminação por chumbo. Esse resultado parece indicar que, ao menos para os meninos, o chumbo é um fator importante nas dificuldades de aprendizagem. Um estudo conduzido por Vega-Dienstmaier, Salinas-Pielago e Gutierrez-Campos (2006) que comparou funções cognitivas de meninos e meninas contaminados por chumbo no Peru encontrou déficits de habilidades cognitivas, especialmente para os meninos.

Carvalho (2001) aponta que as meninas são mais incentivadas a ficar em casa e estudar, enquanto os meninos são incentivados a brincar na rua. Tal contexto pode contribuir para que as meninas tenham um desempenho acadêmico superior. Essa diferença comportamental pode também estar relacionada aos diferentes níveis de contaminação, uma vez que os meninos são mais incentivados a brincar fora de casa, o que contribui para o contato relativamente maior com ambientes contaminados, por exemplo, o solo. Pesquisas futuras poderão considerar este efeito pareando níveis de contaminação e resultados no teste para cada criança.

As diferenças entre gêneros aparecem também em relação à opinião dos pais sobre atenção, memória e rapidez nas tarefas. Esses aspectos podem estar relacionados às questões de estereótipos ligados as diferenças de gênero, uma vez que o conceito em relação às meninas tende a ser superior ao atribuído aos meninos (Carvalho, 2001). Os resultados obtidos no TDE confirmam melhor desempenho acadêmico para as meninas.

Acredita-se que o desempenho inferior dos meninos pode estar relacionado a questões socioculturais tanto no modo de se relacionar com os conteúdos acadêmicos (serem menos incentivados a estudar que as meninas) quanto na maior contaminação por chumbo (brincar mais fora de casa, contato com solo e ambientes contaminados).

Outra hipótese para esses resultados relacionados a diferenças de gênero é que outros determinantes culturais (meninas aprenderem a se organizar, estudar mais, ficar mais em casa) tiveram poder de suprimir e/ou atenuar os efeitos da variável contaminação por chumbo para o grupo feminino.

Tais resultados, embora apontem correlações entre os efeitos do chumbo no desempenho escolar, principalmente para os meninos, devem ser vistos com cautela. Estudos com populações maiores e com grupos de controle deverão ser conduzidos para que esses resultados possam ser confirmados.

\section{Referências}

Araujo, U., Pivetta, F. E., \& Moreira, J.C. (1999). Avaliação da exposição ocupacional ao chumbo: proposta de uma estratégia de monitoramento para prevenção dos efeitos clínicos e sub-clínicos. Cad. Saúde Pública, 15(1), 123-132.

Banks, E. C., Ferretti, L. E., \& Shucard, D. W. (1996). Effects of low level lead exposure on cognitive function in children: a review of behavioral, neuropsychological and biological evidence. Neurotoxicology, 18(1), 237-281.

Bellamy, C. (2004.). Estado mundial de la infancia 2004: las niñas, la educación y el desarrollo. Nueva Yorque: Fondo de las Naciones Unidas para la Infancia.

Bellinger, D.C. (1995). Interpreting the literature on lead and child development: the neglected role of the experimental system. Neurotoxicology and Teratology, 17(3), 201-212.

Bellinger, D.C (2008). Very low lead exposures and children's neurodevelopment Therapeutics and toxicology, 20(2), 172-177.

Benczik, E. B. P. (2000). Transtorno do Déficit de Atenção/ Hiperatividade: Atualização Diagnóstica e Terapêutica. São Paulo: Casa do Psicólogo.

Campana, A.P. (1985). Características antropométricas de escolares e suas relações com o status econômico e o nível intelectual. Tese de Doutorado, Universidade Estadual Paulista, Botucatu-SP.

Capellini, V. L. M. F., Rodrigues, O. M. P. R., Melchiori, L. E., \& Valle, T. G. M. (2008). Crianças contaminadas por chumbo: Estudo comparativo sobre desempenho escolar. Estudos em Avaliação Educacional, 19(39), 155-180.

Capellini, V.L.M.F. (2001). A inclusão de alunos com necessidades educacionais especiais em classes comuns: avaliação do rendimento acadêmico. Dissertação de Mestrado. Universidade Federal de São Carlos, São Carlos-SP.

Carvalho, M,P. (2001). Mau aluno, boa aluna? Como as professoras avaliam meninos e meninas. Estudos Feministas, 9(2), 554-574.

Carvalho, M,P . (2003). Sucesso e fracasso escolar: uma questão de gênero. Educação e Pesquisa, 29(1), 185-193.

Choudhari, R., Sathwara, N. G., Shivgotra, V. K., Patel S., Rathod, R. A., Shaikh, S.M., Shaikh, I., Dodia,S. D., Parikh, J., \& Saiyed, H. N. (2010) Study of lead exposure to children residing near a lead-zinc mine. Indian J Occup Environ Med., 14(2), 58-62. 
Collares, C., \& Moysés.A. (1996). Preconceitos no cotidiano escolar: ensino e medicalização. São Paulo: Cortez .

Committee On Environmental Health (2005). Lead Exposure in Children: Prevention, Detection, and Management. Pediatrics 116(4), 1036-1046.

Correa, J., \& Maclean, M. (1999). Aprendendo a ler e a escrever: a narrativa das crianças sobre alfabetização. Psicologia Reflexão e Crítica, 12(2), 273-286.

Dascânio, D. \& Valle, T. G. M. do. (2007). Avaliação das práticas educativas dos pais de crianças com baixa e alta plumbemia. Pesquisas e Práticas Psicossociais, 2(1), 198-208.

DeBaryshe, B.D., Patterson, G.R., \& Capaldi, D.M. (1993). A performance model for academic achievement in early adolescent boys. Developmental Psychology, 29(5), 795-804.

Diao, B., Wu, Q., \& Zhu, Y. (2011). Impact of chronic lead poisoning on learning and memory in rats. Modern Preventive Medicine, 8, 1417-1418.

Dias, T. L., Enumo, S. R. F., \& Azevedo Junior, R. R. (2004). Influências de um programa de criatividade no desempenho cognitivo e acadêmico de alunos com dificuldade de aprendizagem. Estudos de Psicologia, 9(3), 429-437.

Duncan J.D, Brooks-Gunn, J. \& Klebanov, P.K. (1994). Economic deprivation and early childhood development. Child Development, 65(2), 296-318.

Ginsburg, G.S. \& Bronstein, P. (1993). Family factors related to children's intrinsic/extrinsic motivational orientation and academic performance. Child Development, 64(5), 1461-1474.

He, K., Wang, S. \& Zhang, J. (2009). Blood lead levels of children and its trend in China. Science of The Total Environment, 407(13), 3966-3993.

Instituto Ambiental Vidágua (2002). Poluição por chumbo em Bauru. Recuperado: 01 mai. 2002. Disponível: http://www.vidagua. org.br/chumbo.htm.

Instituto Nacional De Estudos Pedagógicos. (2001). Ministério da Educação e Cultura. Resultados do Saeb 2001. Recuperado: 01 mai. 2002. Disponível: www.inep.gov.br.

Jain, N.B., Laden, F., Guller, U., Shankar, A., Kazani, S., \& Garshick, E. (2005). Relation between blood lead levels and childhood anemia in India. Int J Occup Environ Health, 11(2), 1077-3525.

Jiang, Y., Shi, H., Li, J., Shen, C., Liu, J., \& Yang, H. (2010). Environmental Lead Exposure Among Children in Chengdu,China: Blood Lead Levels and Major Sources. Bull Environ Contam Toxicol, 84, 1-4.
Jorge, M. S., Vitto, L. M., Lamônica, D. A. C., \& Hage, S. R.V. (2008). A exposição ao chumbo como fator de risco para alterações no desenvolvimento da linguagem. Revista Sociedade Brasileira de Fonoaudiologia, 13(2), 161-165.

Macedo, C. S.; Andreucci, L. C., \& Montelli, T.C.B. (2004). Alterações cognitivas em escolares de classe socio-econômica desfavorecida: resultados de intervenção psicopedagógica. Arquivos de NeuroPsiquiatria, 62(3b), 852-857.

Mahmoudian T, Modaresi M, Zarei A, Poursafa P. \& Kelishadi R. (2009). Blood lead levels in children with neurological disorders: a single centre preliminary study, 11(11), 873-876.

Malta, C.G. T., Trigo, L.A.S.C., \& Cunha, L.S. (2000). Saturnismo. Recuperado: 01 set. 2002. Disponível: http://www.geocities.com/ HotSprings/Resort /4486/chumbo1.htm. Acesso em setembro de 2002.

Meira, M. E. M. (2003). Psicologia escolar: Pensamento Crítico e Praticais Profissionais. Em Souza, M. P. R., Tanamachi, E. R., \& Rocha, M. (Orgs.), Psicologia e Educação - desafios teórico práticos. São Paulo: Casa do Psicólogo.

Meireles, H. L. (1981). Direito Municipal Brasileiro. $4^{\mathrm{a}}$ ed. São Paulo: Revista dos Tribunais.

Miranda, M. L.; Maxson,P., \& Kim, D. (2010). Early childhood lead exposure and exceptionality designations for students. International Journal of Child and Adolescent Health, 3(1), 77-84.

Moreira, F.R., \& Moreira, J.C. (2004). Os efeitos do chumbo sobre o organismo humano e seu significado para a saúde. Revista Panamericana de Salud Pública, 15(2), 119-129.

Moszynski, P. (2010). Lead poisoning in Nigeria causes "unprecedented" emergency. BMJ News, 341(1), 4031.

Needleman H.L. (1979). Lead levels and children's psychological performance. New England Journal of Medicine, 301(3), 163.

Needleman, H. (2009). Low Level Lead Exposure: History and Discovery. Anals of epidemiology, 9(4), 235-238.

Needleman, H.L., \& Gatsonis, C. (1990). Low-Level Lead Exposure and the IQ of Children A Meta-analysis of Modern Studies. Jornal da Associação Médica Americana, 263, 673-678.

Smith, M. (1985). Recent work on low level lead exposure and its impact on behavior, intelligence and learning: a revision. Journal of the American Academy of child psychiatry, 24(1), 24-32.

Stein, L.M. (1994). Manual para a aplicação e Interpretação do Teste de Desempenho Escolar (TDE). São Paulo: Casa do Psicólogo. 
Stiles, K., \& Bellinger, D. C. (1993). Neuropsychological correlates of low-level lead exposure in school-age children: a prospective study. Neurotoxicology and Teratology, 15, 27-35.

Thacker S.B, Hoffman D.A, Smith J., Steinberg K., \& Zack M. (1992). Effect of low-level body burdens of lead on the mental development of children. Arch Environ Health, 47(5), 336-346.

Thomson, G.O.B., Raab, G.M., Hepburn, W.S., Hunter, R., Fulton, M.. \& Laxen, D.P.H. (1989). Blood-lead levels and children's behavior: Results from the Edinburgh lead study. Journal of Child Psychology and Psychiatry, 30(4), 515-528.

Tomita, N. E., Padula, N. A. M. R., \& Gepiccb. (2005). Intoxicação por chumbo em crianças e o discurso da imprensa. Ciênc. saúde coletiva, 10(supl.), 111-119.
Vega-Dienstmaier, J. M., Salinas-Pielago, J.E, \& Gutierrez-Campos, M.R. (2006). Níveis de chumbo e funções cognitivas em crianças peruanas. Revista Brasileira de Psiquiatria, 28(1), 33-39.

Warniment, C., Tsang, K., \& Galazka, S.S. (2010). Lead poisoning in children. 81(6), 751-757.

Yule, W. \& Lansdown, R. G. (1983). Blood lead concentrations in school age children, intelligence and attainment in a school population: a pilot study. Devpm. Med. Child Neurol, 23,567-576.

Zhang, X.,MA, R. \& Liu, X. (2010). Shugan Lidan Therapy for Discharging Lead and Antagonizing Damage of Hippocampus in Lead-poisoned Rat. Chinese Journal of Experimental Traditional Medical Formulae, 3.

Recebido em: 14/06/2013

$1^{\text {a. }}$. Reformulaçao em: 19/12/2013

$2^{a}$. Reformulaçao em: 19/06/2014

Aprovado em: 02/07/2014

\section{Sobre as autoras}

\section{Olga Maria Piazentin Rolim Rodrigues}

Doutora em Psicologia Experimental pelo IP/USP/SP, docente do Departamento de Psicologia, da Faculdade de Ciências, da UNESP/Bauru/SP.

\section{Christiana Gonçalves Meira de Almeida}

Doutoranda em Psicologia pela Universidade Federal de São Carlos, docente do curso de Psicologia das Faculdades Integradas de Ourinhos.

Veronica Aparecida Pereira (veronicapereira@ufgd.edu.br)

Doutora em Educação Especial pela UFSCar/São Carlos/SP, docente do Curso de Psicologia, da Faculdade de Ciências Humanas, da Universidade Federal da Grande Dourados, do Mato Grosso do Sul.

\section{Vera Lúcia Messias Fialho Capellini}

Doutora em Educação Especial pela UFSCar/São Carlos/SP, docente do Departamento de Educação, Faculdade de Ciências, da UNESP/ Bauru/SP.

\section{Apoio FAPESP}

O presente estudo é parte de um projeto maior intitulado "Avaliação do desenvolvimento geral e intelectual de crianças de um a 10 anos de idade contaminadas por chumbo", coordenado pela primeira autora. 